\title{
Recurrent and isolated central nervous system blast crisis in chronic myelogenous leukemia
}

\author{
Avnit Sidhu, Sharanjit Singh* \\ Department of Pathology, Armed Forces Medical College, Pune, India
}

\section{Introduction}

Chronic myelogenous leukemia (CML) is the commonest myeloproliferative neoplasm encountered and it is characterized by the hallmark translocation $\mathrm{t}(9 ; 22)$ or BCR-ABL1 transcripts. The natural history of the disease has three phases: the chronic phase (CP) followed by progressive transformation to the accelerated or blast phase. The defining criteria for the blast phase are the presence of at least $20 \%$ blasts in peripheral blood or bone marrow or extramedullary proliferation of blasts [1]. Infiltration of the central nervous system as the sole site of extramedullary blast crisis is exceptional and deserves attention.

\section{Clinical vignette}

We report the case of a 42-year-old male who was diagnosed as a case of chronic myelogenous leukemia in chronic phase (CML-CP) in August 2016. At the time of diagnosis, hemoglobin $(\mathrm{Hb})$ was $11.2 \mathrm{~g} / \mathrm{dL}$, platelet counts were normal, and white blood cell counts were $120 \times 10^{3} / \mu \mathrm{L}$ with left shift, $7 \%$ basophils, and $5 \%$ blasts. Bone marrow cytogenetics confirmed the presence of BCR-ABL1 transcript in all metaphases. Clinically, the patient had splenomegaly $3 \mathrm{~cm}$ below the left costal margin. He was started on imatinib therapy. He responded well to therapy achieving major molecular response (MMR) at the end of 1 year (BCR-ABL1 by reverse-transcriptase polymerase chain reaction $[R T-P C R]=0.015 \%$ ). However, in November 2018 he presented with severe frontal headache and vomiting. Magnetic resonance imaging (MRI) brain revealed chronic small vessel ischemia and nonspecific demyelination. Bone marrow studies showed a normocellular bone marrow. Flow cytometry of cerebrospinal fluid (CSF) was suggestive of pre-B cell lymphoblasts. He was thus diagnosed as CML in central nervous system (CNS) blast crisis and managed with triple intrathecal chemotherapy, which included methotrexate (12 mg), cytosine arabinoside (30 mg), and dexamethasone (4 mg), a total of four doses in a month along with cranial radiotherapy. The patient responded well to the treatment without residual sequelae and was started on the second-generation tyrosine kinase inhibitor (TKI), tablet dasatinib $70 \mathrm{mg}$ twice a day and was being worked up for bone marrow transplantation After 2 months, he again presented with CNS relapse and complained of acute onset ptosis and diplopia in the absence of fever, headache, seizure, or any trauma. Ophthalmologic examination revealed cranial nerve III palsy with pupillary sparing. Haematological parameters were $\mathrm{Hb} 10.0 \mathrm{~g} / \mathrm{dL}$, TLC 3,200/ $/ \mu \mathrm{L}$, and platelet count $177 \times 10^{3} / \mu \mathrm{L}$. Bone marrow study revealed cellular reactive marrow without an increase in blasts. MRI brain showed nonspecific white matter changes. The CSF study showed markedly elevated white blood cell (WBC) count of $270 / \mu \mathrm{L}$ with $90 \%$ blasts (Figure 1); the total protein was $56 \mathrm{mg} / \mathrm{dL}$ with raised globulins, glucose was $55 \mathrm{mg} / \mathrm{dL}$, and adenosine deaminase and lactate dehydrogenase levels were normal. CSF flow cytometry this time again was consistent with pre-B lymphoblasts (positive for CD10, CD19, CD79a, HLA-DR and negative for CD13, CD33, CD117, cyto MP0). He was again started on triple intrathecal chemotherapy and cranial radiotherapy. However, he had progressive deterioration in his neurological status and succumbed to his illness after two cycles of chemotherapy. Postmortem could not be performed due to unwillingness by family members.

\section{Discussion}

Extramedullary blast crisis in CML is rare and CNS involvement is a rarity that is noted in only $5-10 \%$ of patients [2]. Our patient had two episodes of isolated CNS blast crisis, despite having achieved haematologic and molecular
Address for correspondence: Sharanjit Singh,

Department of Pathology, Armed Forces Medical College,

Pune, Maharashtra 411040, India, phone: +94 781231 32,

e-mail: singhsharanjit01@gmail.com

Received: 08.04.2020

Accepted: 17.08 .2020
PTHiT Copyright $\odot 2021$

The Polish Society of Haematologists and Transfusiologists,

Uदि Insitute of Haematology and Transfusion Medicine. 


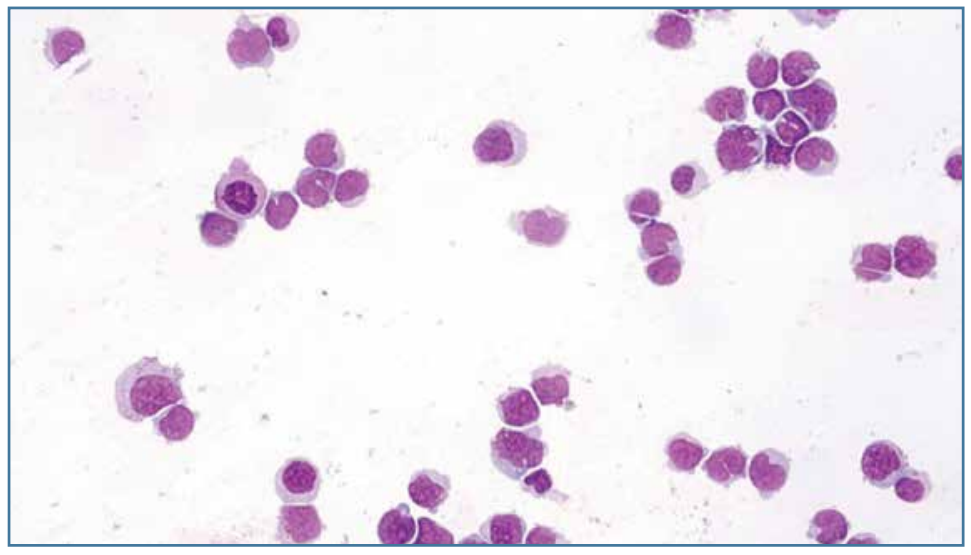

Figure 1. LG stained cytospin smears of cerebrospinal fluid. Markedly cellular smears showing atypical mitosis, numerous blasts which have high N:C ratio, a large nucleus with open chromatin, and prominent nucleoli $400 \times$

remission. The most plausible explanation for extramedullary relapse is the poor penetration of imatinib through the blood-brain barrier, hence CNS serves as the sanctuary site leading to relapses. Second-generation TKls such as dasatinib, nilotinib, and bosutinib are more effective in treating CNS disease due to better penetration through the blood-brain barrier [3]. Unfortunately, our patient relapsed even with dasatinib treatment. Rare case reports of isolated CNS relapse with dasatinib therapy like our patient have been reported $[4,5]$.

Systemic chemotherapy, which includes daily cytarabine $200 \mathrm{mg} / \mathrm{m}^{2}$ and daunorubicin $40 \mathrm{mg} / \mathrm{m}^{2}$ have been used for treatment in CML blast crisis. So far, allogeneic hematopoietic stem-cell transplantation is still the only way to cure CML and is one of the first treatment options for children with chronic myelogenous leukemia [6].

Despite advances in molecular disease detection and monitoring, there is still a need to identify the risk factors leading to CNS relapses. Unfortunately, CSF study is not included in the CML diagnostic workup or monitoring protocols, unlike other acute leukemias and lymphomas.

Through this case, we intend to highlight the fact and also caution physicians that one should be aware of the possibility of CNS relapse in CML patients on imatinib even if they have achieved haematological and cytogenetic remission. Hence, a high index of suspicion, prompt CSF studies, and aggressive management are key to salvaging CML patients.

\section{Authors' contributions}

AS, SS - interpreted patient data regarding the disease; AS - performed the analysis of CSF and flow cytometry and critically revised the manuscript and improved the intellectual content; SS - prepared the draft manuscript.

\section{Conflict of interest}

None.

\section{Financial support \\ None.}

\section{Ethics}

The work described in this article has been carried out in accordance with The Code of Ethics of the World Medical Association (Declaration of Helsinki) for experiments involving humans; EU Directive 2010/63/EU for animal experiments; uniform requirements for manuscripts submitted to biomedical journals.

\section{References}

1. Arber DA, Orazi A, Hasserjian R, et al. The 2016 revision to the World Health Organization classification of myeloid neoplasms and acute leukemia. Blood. 2016; 127(20): 2391-2405, doi: 10.1182/ /blood-2016-03-643544, indexed in Pubmed: 27069254.

2. Rajappa S, Uppin SG, Raghunadharao D, et al. Isolated central nervous system blast crisis in chronic myeloid leukemia. Hematol Oncol. 2004; 22(4): 179-181, doi: 10.1002/hon.737, indexed in Pubmed: 15995975.

3. Kiran PK, Badarke GV, Suresh CN, et al. Isolated central nervous system blast crisis in a case of chronic myeloid leukemia on dasatinib. South Asian J Cancer. 2018; 7(3): 170, doi: 10.4103/sajc. sajc_63_18, indexed in Pubmed: 30112332.

4. Matsuda M, Morita Y, Shimada T, et al. Extramedullary blast crisis derived from 2 different clones in the central nervous system and neck during complete cytogenetic remission of chronic myelogenous leukemia treated with imatinib mesylate. Int J Hematol. 2005; 81(4): 307-309, doi: 10.1532/IJH97.04188, indexed in Pubmed: 15914360.

5. Juneja R, Dhamija G, Dadu T, et al. Biphenotypic extramedullary blast crisis with MLL gene rearrangement in a case of chronic myeloid leukemia following dasatinib therapy: an unusual case. Indian J Pathol Microbiol. 2016; 59(2): 259-260, doi: 10.4103/0377-4929.182031, indexed in Pubmed: 27166066.

6. Jin M, Xuan C, Gao J, et al. Chronic myeloid leukemia extramedullary blast crisis presenting as central nervous system leukemia: a case report. Medicine (Baltimore). 2018; 97(45): e13131, doi: 10.1097/ /MD.0000000000013131, indexed in Pubmed: 30407337. 